\title{
Interplay of COVID-19 and cardiovascular diseases in Africa: an observational snapshot
}

\author{
Graham Chakafana $^{1,2} \cdot$ Daniel Mutithu ${ }^{1,2} \cdot$ Julian Hoevelmann ${ }^{1,4} \cdot$ Ntobeko Ntusi $^{1,2,3} \cdot$ Karen Sliwa $^{1,2}$
}

Received: 29 June 2020 / Accepted: 23 July 2020 / Published online: 18 August 2020

c) Springer-Verlag GmbH Germany, part of Springer Nature 2020

\begin{abstract}
The COVID-19 pandemic, which started around December 2019 has, at present, resulted in over 450,000 deaths globally, and approximately $1 \%$ of these deaths have been reported in Africa. Despite the high prevalence of COVID-19 risk factors, namely: hypertension, diabetes, chronic pulmonary disease, cardiovascular diseases (CVDs) such as rheumatic heart disease, compromised immunity and obesity, low case fatality rates have been recorded in many parts of Africa so far. COVID-19 severity has previously been shown to be worse in patients with CVD and hypertension. We observed the severity of COVID19 and mortality rates in Africa, and compared outcomes with prevalence of established risk factors (hypertension and CVD). We stratified data as per the United Nations' 5 African subregions. North African countries show a positive association between the risk factors and the mortality rates from COVID-19. However, we observed discordant patterns in the relationship between COVID-19, and either CVD or hypertension, in sub-Saharan African countries. In this paper, we also review the pathogenesis of SARS-CoV-2 infection and how it worsens CVD and postulate that the differences in modulation of the renin-angiotensin-aldosterone system (RAAS) axis which controls angiotensin-converting enzyme (ACE)/ACE2 balance may be an important determinant of COVID-19 outcomes in Africa.
\end{abstract}

Keywords COVID-19 $\cdot$ SARS-CoV-2 $\cdot$ Cardiovascular diseases $\cdot$ Hypertension $\cdot$ Africa $\cdot$ RAAS

\section{Introduction}

The novel severe acute respiratory syndrome coronavirus 2 (SARS-CoV-2) is the causative agent for the global COVID19 pandemic [1]. Although there has been an increase in

Electronic supplementary material The online version of this article (https://doi.org/10.1007/s00392-020-01720-y) contains supplementary material, which is available to authorized users.

Karen Sliwa

karen.sliwa-hahnle@uct.ac.za

1 Hatter Institute for Cardiovascular Research in Africa, Faculty of Health Sciences, University of Cape Town, Cape Town, RSA

2 Division of Cardiology, Department of Medicine, Faculty of Health Sciences, University of Cape Town, Cape Town, RSA

3 Cape Universities Body Imaging Centre, Faculty of Health Sciences, University of Cape Town, Cape Town, RSA

4 Klinik für Innere Medizin III - Kardiologie, Angiologie Und Internistische Intensivmedizin, Universitätsklinikum Des Saarlandes, Homburg (Saar), Deutschland the number of new COVID-19 cases globally, Africa has surprisingly low infection and mortality rates. While SARSCoV-2 infection mostly presents with mild symptoms, a small proportion of patients may develop a severe clinical picture characterised by acute respiratory distress syndrome (ARDS) and/or angina (Fig. 1) [2]. Crucial aspects of host-pathogen interactions and mechanisms of systemic complications remain speculative. Of interest are the cardiotoxic effects of COVID-19 and how these impact on disease severity. There is an urgent need to understand SARS-CoV-2 pathophysiology and associated cardiac complications. Given that cardiovascular diseases (CVDs) have emerged as a major predictor of COVID-19 severity [3], we sought to investigate this correlation within the African context. In addition, we report possible cardiovascular complications associated with COVID-19 and pathophysiological differences that may arise within various African regions.

The virus spike (S) protein binds to the host's angiotensin-converting enzyme 2 (ACE2) receptor allowing for viral entry into cells (Fig. 1). ACE2 is a membrane-bound aminopeptidase which is highly expressed in the heart and, to a lesser extent, in lung tissue [4]. It is well established 
A

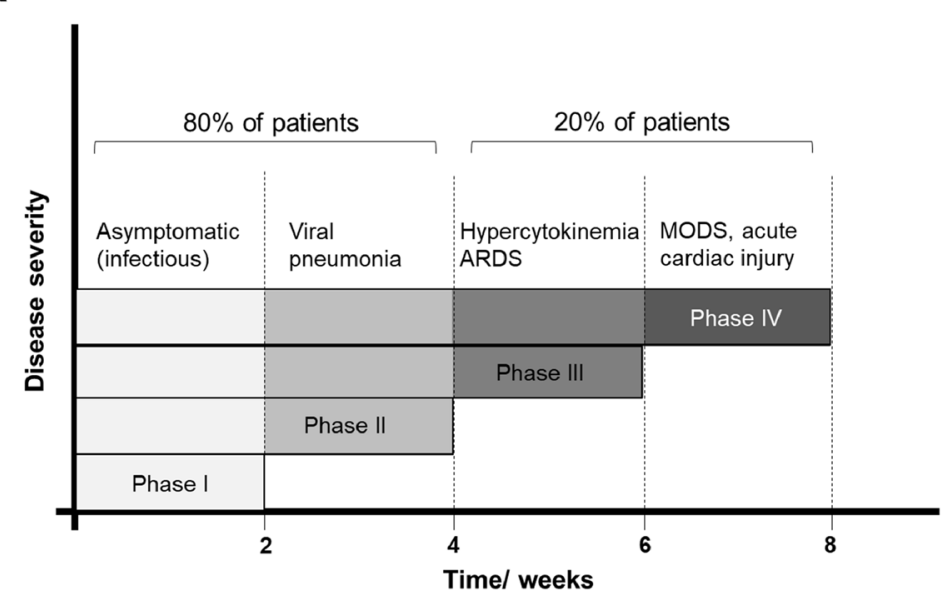

Eyes

1. Rod \& cone photoreceptor cells

2. Muller glial cells

B

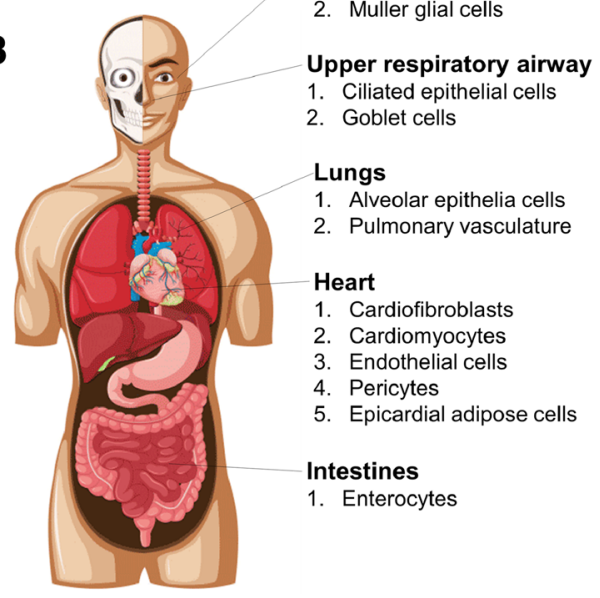

C

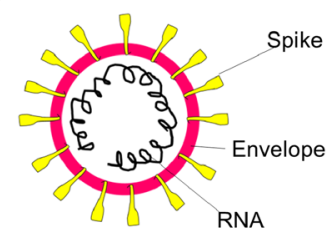

SARS Cov-2

pike (S) glycoprotein
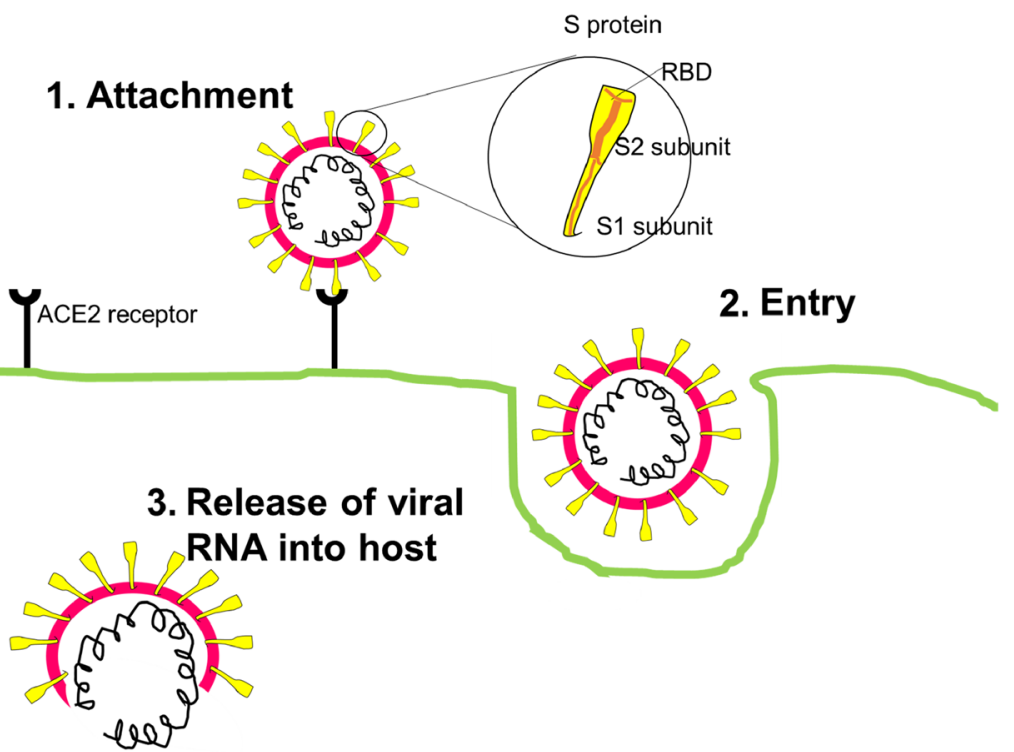

4. Protein synthesis
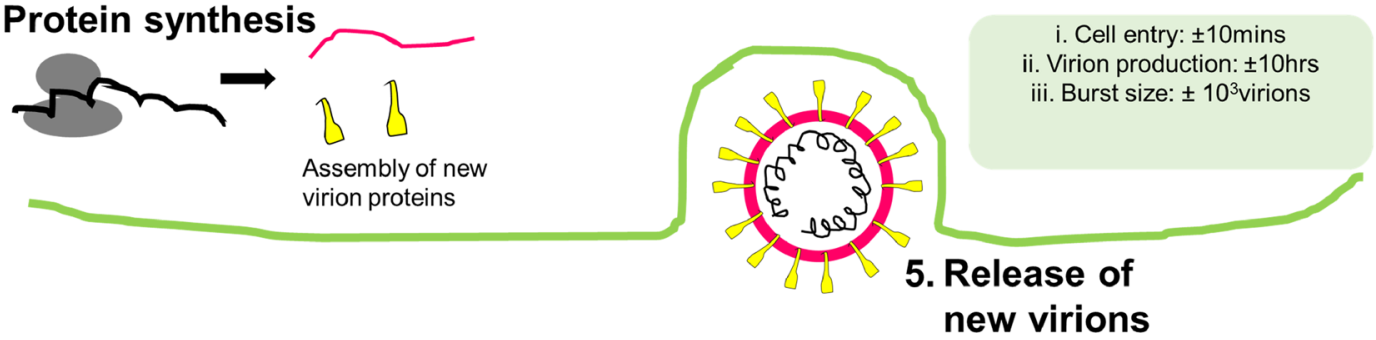

Fig. 1 Clinical presentation of COVID-19 and SARS Cov-2 infection. a The clinical progression of COVID-19 occurs in four distinct phases that are characterised by various symptoms as shown Adapted from Oberfeld B et al. SnapShot: COVID-19. Cell. 2020;181(4):954e1. b The organs in which ACE2 receptors are expressed include the eyes, respiratory airway tract, lung, heart, and small intestines. The respiratory tract is the primary site of ACE2 infection. c The infec- tion cycle of SARS-CoV-2 is initiated when the virion attached to the hosts ACE2 receptor protein (1). Upon attachment, the virion then enters the cells via endocytosis (2) and the viral RNA is released in the host cells (3). Using the host cells' protein synthesis machinery, new viral proteins are synthesized and assembled; therefore, allowing for multiplication. (4). The new viruses are then released from the host cell (5) 
from experimental studies that coronavirus infection can cause myocarditis, cardiomyopathy and congestive heart failure [5]. COVID-19 may directly or indirectly trigger cardiovascular complications (Fig. 2). A recent study demonstrated that COVID-19 can induce cardiac damage in individuals without any underlying CVD [6]. SARS$\mathrm{CoV}-2$ infection can indirectly induce myocardial injury due to a cytokine storm triggered by viral infection, which may lead to increased vascular wall permeability accompanied by myocardial oedema [7]. Furthermore, the virus causes inflammation and fluid retention in the alveoli, which then leads to hypoxia and subsequently impacts on cardiac output [8]. Ultimately, this may trigger myocardial injury, decompensated heart failure and increased risk of arrhythmias in patients with pre-existing CVD [9].

COVID-19 may also directly induce pathological changes in the heart tissue due to viral replication within the myocardium $[10,11]$. Indeed, case studies on autopsy and endomyocardial biopsy reports showed evidence for an acute lymphocytic myocarditis, which may aggravate to inflammatory cardiomyopathy $[8,12,13]$. There is a unique interplay between COVID-19 and CVD. Interestingly, effects of COVID-19 display unique variations across different populations and subgroups. For instance, COVID-19 infection and mortality rates in Africa are lower than those observed globally [14]. While this may reflect evolving population dynamics from infection acquired later, the possibility of

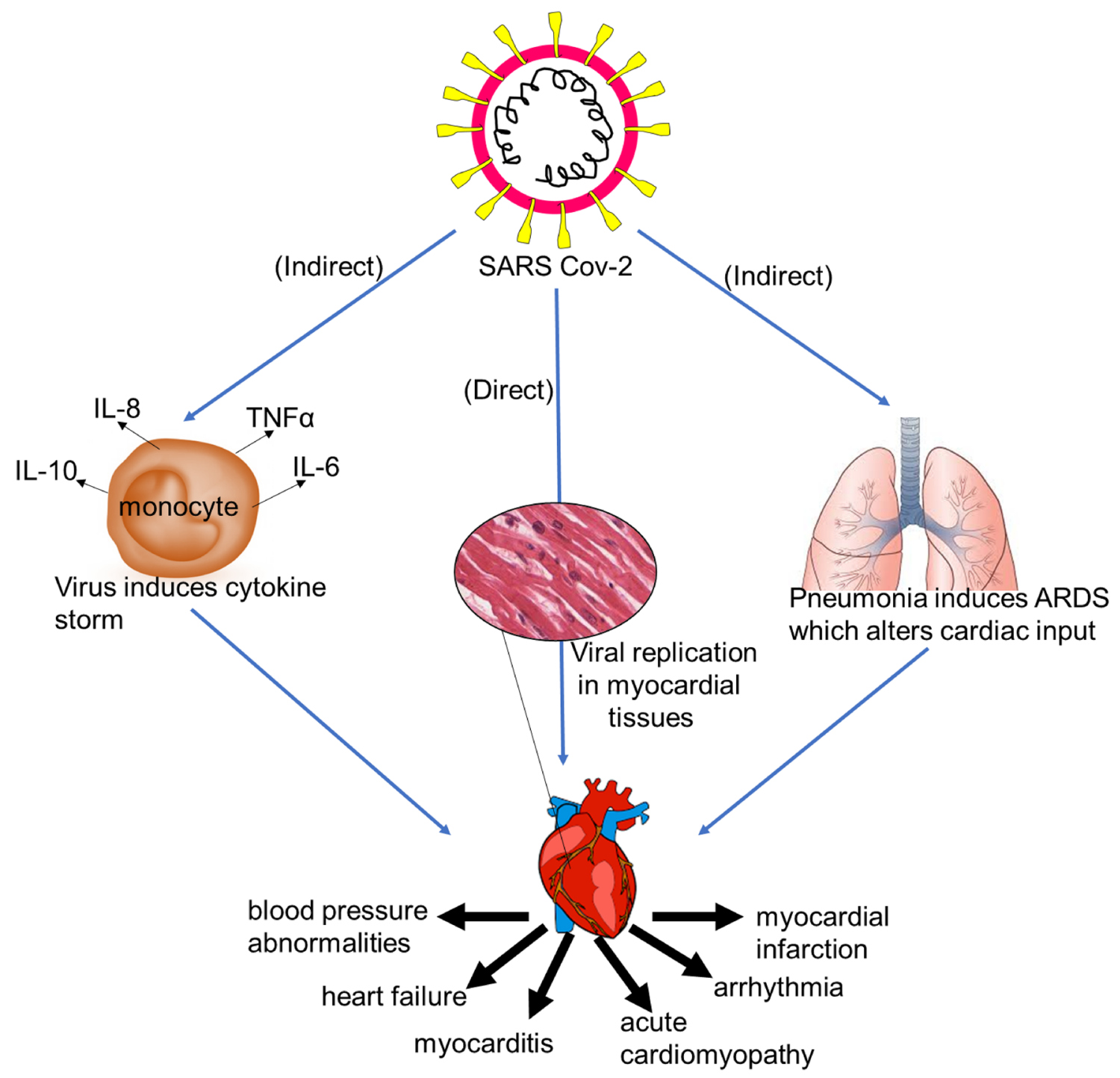

Fig. 2 Cardiotoxic effects of SAR Cov-2 infection. The cardiotoxic effects of SARS-CoV-2 may either be direct or indirect. SARS-CoV-2 may directly affect the heart through direct viral replication within the myocardium, thus resulting in myocardial damage. Alternatively, SARS-CoV-2 infection may indirectly induce myocardial inflammation which impairs cardiac function 
genetic differences or other factors that may predispose some individuals to exaggerated immune responses and adverse cytokine storm exists. We therefore sought to understand the correlation between CVD and COVID-19 in Africa.

\section{Epidemiology of COVID-19 infection in Africa}

On 23 June, a cumulative total of 321,885 confirmed COVID-19 cases have been reported on the African continent. In total, there were 8481 COVID-19-related deaths (case fatality ratio $2.6 \%$ ) and 153,023 cases were reported to have recovered. The highest number of cases in the WHO African Region had been reported from 10 countries: South Africa (106,108), Nigeria (21,371), Ghana (14,568), Algeria $(12,076)$, Cameroon (12,041), Côte d'Ivoire (7904), Senegal (6034), Democratic Republic of Congo (6027), Guinea (5104) and Kenya (4952). The highest numbers of deaths were reported from South Africa 2102 (40\%), Algeria 861 (16\%), Nigeria 533 (10\%), Cameroon 308 (5.9\%), accounting for $74 \%$ of the total deaths reported in the region. The highest case fatality ratios were observed in Chad (8.6\%), Algeria (7.1\%), Niger (6.4), Burkina Faso (5.9\%), Mali (5.6), Angola (5.3\%) and Liberia (5.2\%). As of 23 June 2020, the 7 African countries in the WHO EMRO Region reported a total of 84976 confirmed COVID-19 cases: Egypt (56 809), Morocco (10,264), Sudan (8698), Djibouti (4617), Somalia (2835), Tunisia (1159) and Libya (594). In addition, a total of 3224 deaths have been recorded from Egypt (2278), Sudan (533), Morocco (214), Somalia (90), Tunisia (50), Djibouti (49) and Libya (10). Health workers have been significantly affected by COVID-19, with 5824 being infected in 37 countries since the beginning of the outbreak, and South Africa being most affected with 2084 infected health workers [15]. The highest increase in positive cases are currently seen for the southern African region.

\section{COVID-19 and CVD interplay: Africa}

Africa is a major contributor to the global burden of CVD [16]. The advent of COVID-19 infection exacerbates cardiac failure symptoms in patients with pre-existing cardiovascular diseases and results in adverse outcome of COVID-19 in these patients [8]. Despite the high prevalence of CVDs in Africa, there has so far been a low COVID-19 case fatality in Africa. Recent reports have suggested the high use of antiretroviral therapy for management of HIV infection or the widespread Bacille Calmette-Guerin (BCG) vaccination (mandatory in Africa) as possible explanations for these low COVID-19 mortality rates [17]. However, we have found no correlation between BCG vaccination and COVID-19 mortality rates in Africa (Supplementary Table S1). For instance, Algeria, which has an average BCG vaccination coverage of $>70 \%$ over the past 50 years had a high COVID19 mortality of about $7.12 \%$. On the contrary, Nigeria which only started a national immunisation programme in 1985, had a relatively low COVID-19 mortality rate of approximately $2.57 \%$, though this may also reflect ascertainment bias (Supplementary Table S1).

We specifically compared Algeria and Nigeria which both display comparable hypertension and CVD prevalence rates. However, Nigeria showed a three times higher mortality due to COVID-19 than Algeria (Table 1). This suggests that factors other than cardiovascular-related risk factors may contribute to these observed effects of the virus within the different populations. Of note, Algeria has a much older population than Nigeria. A Chinese study involving 44,000 confirmed cases revealed that the highest case fatality rates were observed in patients over 60 years of age [18]. It is conceivable that the generally lower life expectancy of Africans, and not the prevalence of CVD, may account for the reduced severity of COVID-19 infections. Indeed, ACE2 expression has also been demonstrated to be lower in older individuals, thus predicting a more severe cytokine storm in older individuals [19]. In addition, differences observed between the two populations may also hint at possible genetic variations accounting for differences in vulnerability to COVID-19.

We also conducted comparisons between Egypt and South Africa. Although both countries have a high number of positive cases in the population, South Africa shows a lower mortality rate (Table 2). While South Africa has a higher prevalence of hypertension than Egypt which would, theoretically, predict worse outcomes, we observed lower COVID-19 mortality rates in South Africa. Furthermore, we also observed higher COVID-19 mortality rates in Egypt than in Ghana, although both countries exhibit comparable hypertension prevalence and CVD mortality burden. From these observations, it appears that there is no direct relationship between COVID-19 mortality rates and CVD burden in SSA. SARS-CoV-2 infection outcomes vary across the different populations in SSA. The Egyptian population is predominantly Arabic, while the South African population is predominantly composed of black Africans. Nonetheless, all the African countries still exhibited far lower mortality rates than the USA and UK (Tables 1 and 2).

\section{Impact of hypertension on COVID-19 severity in Africa}

Prevalence of uncontrolled hypertension (17-70\%), which steeply increases with age, is high in Africa [16, 20]. The involvement of the renin-angiotensin-aldosterone system (RAAS) in the pathogenesis of hypertension and upregulation of the immune system through the ACE/AngII/ $\mathrm{AT}_{1}$ axis is irrefutable [21]. ACE converts AngI to AngII, which has been shown to mediate pro-inflammatory effects 
A

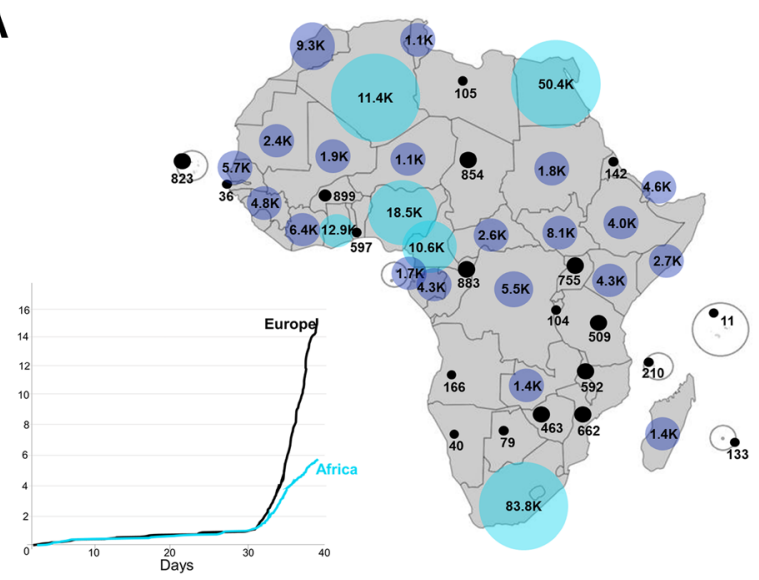

B

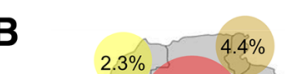

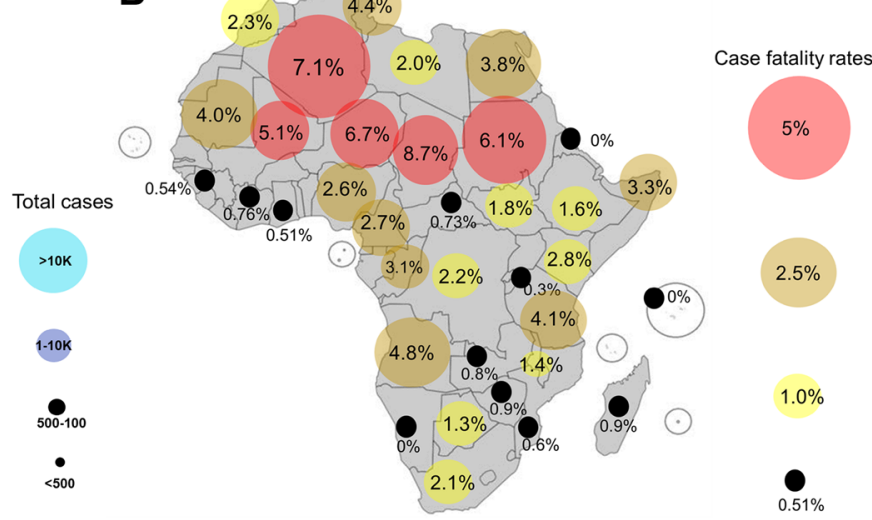

C
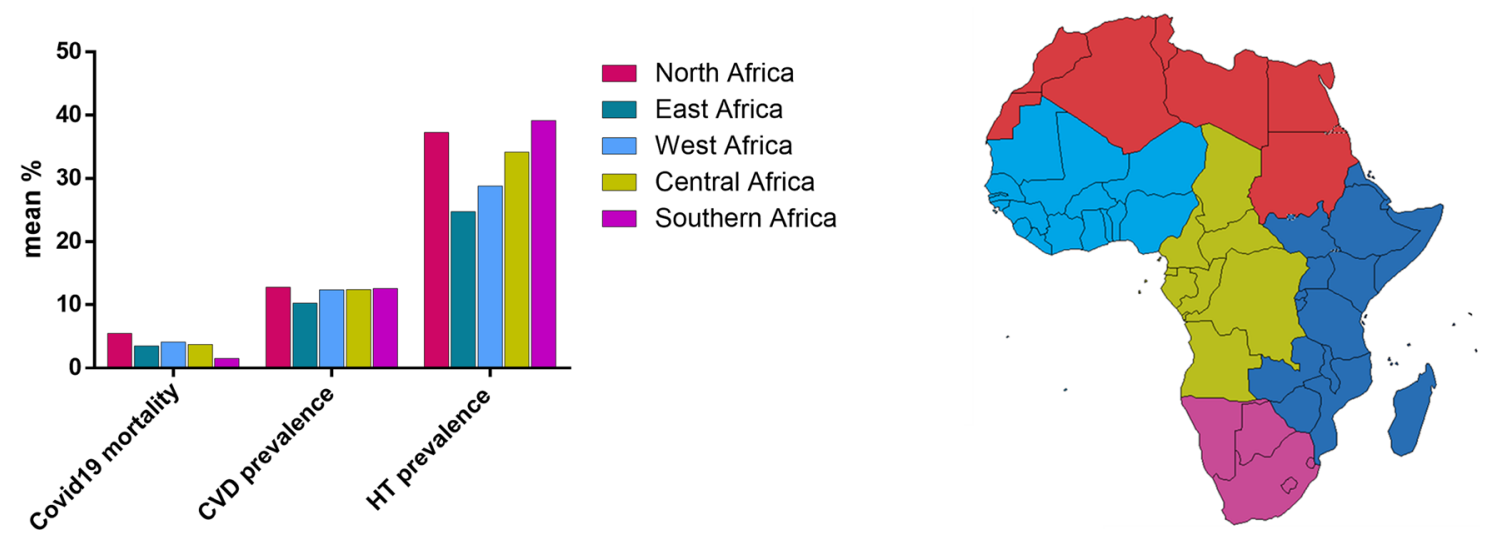

Fig. 3 The overview of COVID19 mortality, CVD mortality burden, and hypertension prevalence in Africa. COVID19 cases in Africa show varied distribution across the continent (a). The insert shows that Africa had a relatively lower rise in cases. A map of Africa showing COVID-19 mortality rates* (b). Comparing the COVID-19

Table 1 Comparison of COVID19 in Algeria and Nigeria

\begin{tabular}{llll}
\hline & Algeria & Nigeria & UK \\
\hline Positive cases* & 11,385 & 18,480 & 303,110 \\
Deaths* $^{*}$ & 811 & 475 & 42,589 \\
Mortality rate* $_{\text {CVD mortality burden }}^{*}$ & 7.12 & 2.57 & 14.1 \\
Hypertension prevalence & 14.0 & 12.0 & 4.0 \\
BCG commencement year & 35.0 & 36.5 & 30.0 \\
Population size & 1935 & 1921 & - \\
Life expectancy & $39.7 \mathrm{M}$ & $182.2 \mathrm{M}$ & $52.8 \mathrm{M}$ \\
Poverty & 74.8 & 52.8 & 81.2 \\
\hline
\end{tabular}

*COVID-19 data as at 20 June 2020 obtained from https://ourworldin data.org/covid-testing\#source-information-country-by-country. ${ }^{\ddagger}$ Indicates probability of deaths due to CVD between ages 30-70 years as at 2012. Data source: WHF mortalities and hypertension, and CVD seemed directly proportional to COVID-19 mortality rates in North Africa. However, there seems to be discordant patterns with the rest of the continent (c). *COVID19 Data as at 20 June 2020 sourced from https://ourworldindata.org/ covid-testing\#source-information-country-by-country

Table 2 Comparison of COVID19 in South Africa and Egypt

\begin{tabular}{lllll}
\hline & South Africa & Ghana & Egypt & USA \\
\hline Positive cases* & 83,890 & 12,929 & 50,437 & $1,680,400$ \\
Deaths* & 1,737 & 66 & 1,938 & 99,130 \\
Mortality rate* & 2.07 & 0.51 & 3.84 & 18.0 \\
CVD mortality burden & 14.0 & 8.00 & 16.0 & 6.0 \\
Hypertension prevalence & 53.6 & 28.0 & 26.30 & 29.0 \\
BCG commencement & 1974 & 1978 & 1974 & - \\
$\quad$ & & & \\
year & $57.2 \mathrm{M}$ & $53.5 \mathrm{M}$ & $91.5 \mathrm{M}$ & $328 \mathrm{M}$ \\
Life expectancy & 57.2 & 49.0 & 71.1 & 78.5 \\
Poverty & $53 \%$ & $46 \%$ & $25 \%$ & $12 \%$ \\
\hline
\end{tabular}

*COVID-19 data as at 20 June 2020 obtained from https://ourworldin data.org/covid-testing\#source-information-country-by-country. ${ }^{\ddagger}$ Indicates probability of deaths due to CVD between ages 30-70 years as at 2012. Data source: WHF 
after binding the $\mathrm{AT}_{1}$ receptors [21, 22]. Accumulation of AngII induces vasoconstriction, elevated sodium retention and increased blood pressure (Fig. 4). Moreover, RAAS through ACE/AngII/AT ${ }_{1}$ R and ACE2/Ang-(1-7)/MAS axes regulate immune response. In addition, AngII is associated with increased expression of IL-12, nitric oxide and TNF- $\alpha$, which promote an exacerbated immune response [21-23].

The anti-inflammatory activity of the ACE2 is driven by Ang-(1-7) through activation of the MAS receptor, as well as inhibition of the NF-kB and activation of Nrf-2 pathways [24]. There is a need for an intricate balance between ACE/ AngII/ $\mathrm{AT}_{1} \mathrm{R}$ and the ACE2/Ang-(1-7)/MAS axes. Reduced expression of ACE2 due to SARS-CoV-2 infection alters the balance, leading to activation of the ACE pro-inflammatory axis, [25-27] (Fig. 5).

It is worth noting that the regimen of ACE inhibitors commonly used in the African population are marred with contraindications which neither pharmacogenomics nor genetics seem to offer a solid explanation for [28, 29]. In as much as ACE/ACE2 axes plays an important role in hypertensives of African descent, further research should be done to investigate potential involvement of other molecules in the RAAS complex [30]. Potential involvement of other hypertensive molecules that are not directly controlled by the ACE/ACE2 inflammatory pathways would potentially explain the disproportionate mortalities, despite high prevalence of hypertensives in African populations.

Despite COVID-19 infection suppressing ACE2 expression there is no upregulation of ACE-mediated acute lung injury (ALI) even in hypertensives with an unchecked ACE/ACE2 axis (Fig. 5). Further, ACE2 expression levels decrease with age and SSA's population is younger than that of North African countries that showed high SARS-CoV-2 infection-related mortalities (Fig. 3). In younger people, following SARS-CoV-2 infection, ACE2 protection is not totally lost and hence patients do not present with ARDS as frequently [31,32]. We recommend further research into the role of the RAAS complex components within the African hypertensive population to define better therapeutic targets. Further, based on our observations it is prudent that COVID19 patients of the African population should be continued on their antihypertensive therapy.

\section{Genetics as a determining factor for COVID-19 cardiotoxic effects}

Although SARS-CoV-2 results in different outcomes in various populations, there are few reports on the role of host genetics in COVID-19 susceptibility and severity. Genetic variations affecting the RAAS axis may result in different clinical outcomes of SARS-CoV-2 infection. Unique associations of the ACE gene polymorphisms with regulation of serum ACE activity in white and black children have been reported in previous studies [33]. ACE (D/I) polymorphisms in intron 16, which is associated with alterations in circulating and tissue concentrations of ACE, have also been reported [34]. This D/I polymorphism shows geographical variation and distribution which may account for

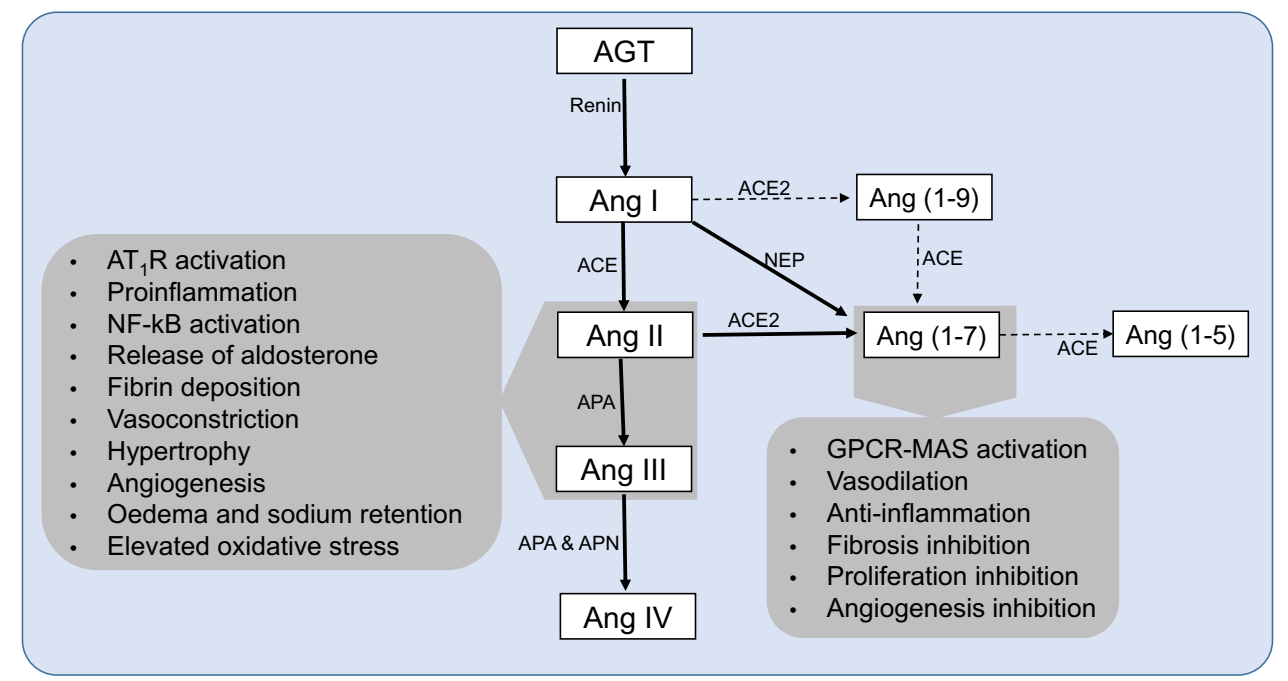

Fig. 4 Schematic summary of Renin-angiotensin system showing the main pathways associated with cardiovascular and immunology pathologies. Angiotensinogen (AGT) is cleaved by renin from the kidneys into angiotensin I (Ang I) which is then converted to Ang II by angiotensin converting (ACE), which is associated with most of the pathologies. Ang II is also hydrolyzed to a lesser extent by neutral endopeptidase (NEP) to Ang-(1-7). Further, Ang II is hydrolyzed to Ang III by aminopeptidase A which is later hydrolyzed to Ang IV by amino peptidase N. Ang I to a lesser extent is converted to Ang(1-9) by angiotensin converting enzyme 2 (ACE2) which is further hydrolyzed to Ang-(1-7) by ACE. The major activity for ACE2 is hydrolyzing Ang II to Ang-(1-7) which associated with cell survival activities 


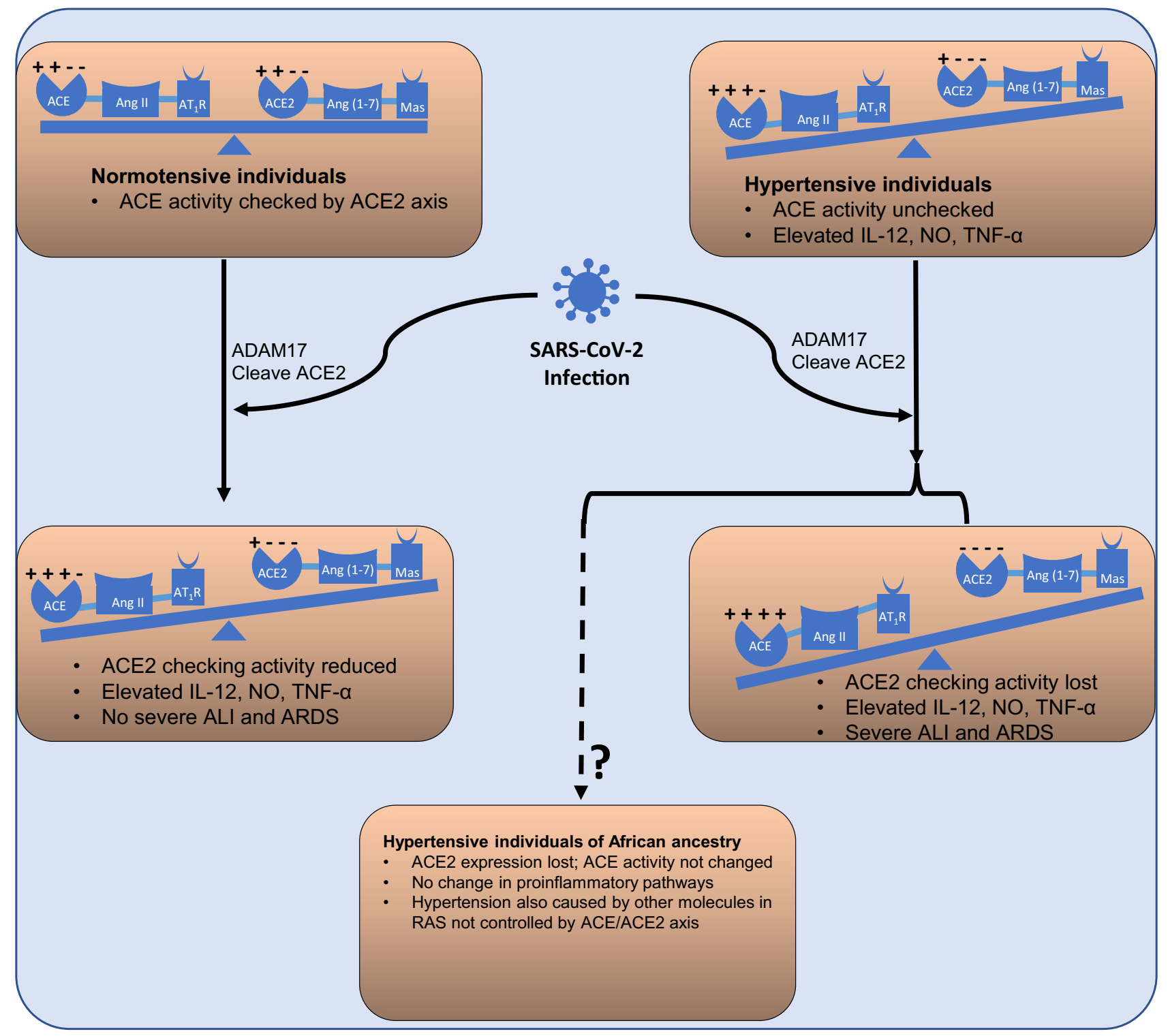

Fig. 5 Schematic summary of the ACE/ACE2 axes balance in normotensive and hypertensive individuals and the role it plays in developing mild or severe ALI and ARDS upon SARS-CoV-2 infection. The proposed mechanism further demonstrates how the ACE/ACE2 axis may not be directly involved in hypertensive patients of African

variable COVID-19 mortality rates in African countries [34]. In another study, a significant correlation was noted between COVID-19 mortality and the prevalence of the ACE1 D-allele in European countries. The D allele is associated with a reduced expression of ACE2 thereby potentially altering the ACE/ACE2 axis in hypertensive patients [35]. Recent studies have indicated high allelic frequencies in the eQTL variants that are associated with higher ACE2 expression in some East Asian populations [36, 37]. Although there is still paucity of data on genetic variations, the discordant patterns in COVID-19 and hypertension in ancestry upon COVID-19 infection. ALI Acute Lung Injury, ARDS Acute Respiratory distress syndrome, $I L-12$ Interleukin 12 , NO nitric oxide, TNF- $a$ Tumor necrosis factor alpha, ACE and ACE2 Angiotensin Converting enzyme and angiotensin converting enzyme 2

Africa may possibly stem from genetic variations within the diverse ethnic and racial populations.

\section{Conclusion}

We observed an indiscriminate prevalence of SARS-CoV-2 infection in Africa. ACE2 expression and distribution are key determinants for SARS-CoV-2 infection. In addition, ACE2 expression is higher in the heart than it is in the lungs. Reports have also indicated that patients suffering from heart 
failure have a higher expression of ACE2, as compared to healthy individuals [38]. Globally, high CVD and hypertension prevalence were directly associated with high COVID19 infection-related mortalities. While observations in North African countries are concordant with this notion, discordant patterns within SSA populations were observed. There may be differences in pathophysiology in different populations with upon SARS-Cov-2 infection. As ACE/ACE2 axis is modulated differently within individuals, we propose that the disparate patterns between COVID-19 mortality rates and CVD prevalence may be a result of differences in ACE/ ACE2 modulation. This will, however, require further experimental validation and provide a platform for targeted drug therapy.

Authors' contributions GC and DM drafted the manuscript, KS and $\mathrm{NN}$ funded and reviewed the manuscript and $\mathrm{JH}$ reviewed the final draft. All authors agreed on the final manuscript for submission.

Funding GC is a recipient of the UCT Faculty of Health Sciences, URC grant. DM is funded through Blue Skies research grant from the National Research Foundation [NRF] (Grant Numbers 104839 and 105858) and Lily and Ernst Hausmann Trust. NN gratefully acknowledges support from the South African Medical Research Council, the NRF and the Lily and Ernst Hausmann Trust.

\section{Compliance with ethical standards}

Conflicts of interest The authors have no conflicting interests to disclose.

\section{References}

1. Yuki K, Fujiogi M, Koutsogiannaki S (2020) COVID-19 pathophysiology: a review. Clin Immunol 215:108427

2. World Health Organization (2020) Coronavirus disease 2019 (COVID-19): situation report, 73. World Health Organization, Geneva

3. Rath D, Petersen-Uribe A, Avdiu A, Witzel K, Jaeger P, Zdanyte $M$ et al (2020) Impaired cardiac function is associated with mortality in patients with acute COVID-19 infection. Clin Res Cardiol. https://doi.org/10.1007/s00392-020-01683-0

4. Li G, Hu R, Zhang X (2020) Antihypertensive treatment with ACEI/ARB of patients with COVID-19 complicated by hypertension. Hypertens Res 43(6):588-590

5. Edwards S, Small JD, Geratz JD, Alexander LK, Baric RS (1992) An experimental model for myocarditis and congestive heart failure after rabbit coronavirus infection. J Infect Dis 165(1):134-140

6. Madjid M, Safavi-Naeini P, Solomon SD, Vardeny O (2020) Potential effects of coronaviruses on the cardiovascular system: a review. JAMA Cardiol. https://doi.org/10.1001/jamacardio .2020 .1286

7. Tay MZ, Poh CM, Renia L, MacAry PA, Ng LFP (2020) The trinity of COVID-19: immunity, inflammation and intervention. Nat Rev Immunol 20(6):363-374

8. Paramasivam A, Priyadharsini JV, Raghunandhakumar S, Elumalai P (2020) A novel COVID-19 and its effects on cardiovascular disease. Hypertens Res 43(7):729-730
9. Li B, Yang J, Zhao F, Zhi L, Wang X, Liu L et al (2020) Prevalence and impact of cardiovascular metabolic diseases on COVID19 in China. Clin Res Cardiol 109(5):531-538

10. Bohm M, Frey N, Giannitsis E, Sliwa K (2019) Coronavirus Disease (COVID-19) and its implications for cardiovascular care: expert document from the German Cardiac Society and the World Heart Federation. Clin Res Cardiol. https://doi.org/10.1007/s0039 2-020-01656-3

11. Guzik TJ, Mohiddin SA, Dimarco A, Patel V, Savvatis K, MarelliBerg FM et al (2020) COVID-19 and the cardiovascular system: implications for risk assessment, diagnosis, and treatment options. Cardiovasc Res. https://doi.org/10.1093/cvr/cvaa106

12. Sala S, Peretto G, Gramegna M, Palmisano A, Villatore A, Vignale D et al (2020) Acute myocarditis presenting as a reverse Tako-Tsubo syndrome in a patient with SARS-CoV-2 respiratory infection. Eur Heart J 41(19): 1861-1862

13. Yao XH, Li TY, He ZC, Ping YF, Liu HW, Yu SC et al (2020) A pathological report of three COVID-19 cases by minimal invasive autopsies. Zhonghua Bing Li Xue Za Zhi 49(5):411-417

14. Nachega JB, Seydi M, Zumla A (2020) The late arrival of COVID19 in Africa - mitigating pan-continental spread. Clin Infect Dis. https://doi.org/10.1093/cid/ciaa353

15. World Health Organization (2020) COVID-19 Situation update for the WHO African region 24 June 2020 External Situation Report 17. World Health Organization, Geneva

16. Keates AK, Mocumbi AO, Ntsekhe M, Sliwa K, Stewart S (2017) Cardiovascular disease in Africa: epidemiological profile and challenges. Nat Rev Cardiol 14(5):273-293

17. Miller A, Reandelar MJ, Fasciglione K, Roumenova V, Li Y, Otazu GH (2020) Correlation between universal BCG vaccination policy and reduced morbidity and mortality for COVID-19: an epidemiological study. medRxiv. https://doi.org/10.1101/2020.03.24.20042 937

18. Wu A, Peng Y, Huang B, Ding X, Wang X, Niu P et al (2020) Genome composition and divergence of the novel coronavirus (2019-nCoV) originating in China. Cell Host Microbe 27(3):325-328

19. Li MY, Li L, Zhang Y, Wang XS (2020) Expression of the SARSCoV-2 cell receptor gene ACE2 in a wide variety of human tissues. Infect Dis Poverty 9(1):45

20. Bosu WK, Reilly ST, Aheto JMK, Zucchelli E (2019) Hypertension in older adults in Africa: a systematic review and metaanalysis. PLoS ONE 14(4):e0214934

21. Crowley SD, Rudemiller NP (2017) Immunologic effects of the renin-angiotensin system. J Am Soc Nephrol 28(5):1350-1361

22. Gaddam RR, Chambers S, Bhatia M (2014) ACE and ACE2 in inflammation: a tale of two enzymes. Inflamm Allergy Drug Targets 13(4):224-234

23. de Leeuw PW (1999) How do angiotensin II receptor antagonists affect blood pressure? AmJ Cardiol 84(2):5-6

24. Fang Y, Gao F, Liu Z (2019) Angiotensin-converting enzyme 2 attenuates inflammatory response and oxidative stress in hyperoxic lung injury by regulating NF-kappaB and Nrf2 pathways. QJM 112(12):914-924

25. Hu HH, Zhang RF, Dong LL, Chen EG, Ying KJ (2020) Overexpression of ACE2 prevents hypoxia-induced pulmonary hypertension in rats by inhibiting proliferation and immigration of PASMCs. Eur Rev Med Pharmacol Sci 24(7):3968-3980

26. Guignabert C, de Man F, Lombes M (2018) ACE2 as therapy for pulmonary arterial hypertension: the good outweighs the bad. Eur Respir J 51:6

27. Tikellis C, Thomas MC (2012) Angiotensin-converting enzyme 2 (ACE2) Is a key modulator of the renin angiotensin system in health and disease. Int J Pept 2012:256294

28. Brewster LM, Seedat YK (2013) Why do hypertensive patients of African ancestry respond better to calcium blockers and diuretics 
than to ACE inhibitors and beta-adrenergic blockers? A systematic review. BMC Med 11:141

29. Brewster LM, van Montfrans GA, Oehlers GP, Seedat YK (2016) Systematic review: antihypertensive drug therapy in patients of African and South Asian ethnicity. Intern Emerg Med 11(3):355-374

30. Cohall D, Ojeh N, Ferrario CM, Adams OP, Nunez-Smith M (2020) Is hypertension in African-descent populations contributed to by an imbalance in the activities of the ACE2/Ang-(1-7)/Mas and the ACE/Ang II/AT1 axes? J Renin Angiotensin Aldosterone Syst 21(1):1470320320908186

31. Yoon HE, Kim EN, Kim MY, Lim JH, Jang IA, Ban TH et al (2016) Age-associated changes in the vascular renin-angiotensin system in mice. Oxid Med Cell Longev 2016:6731093

32. Schouten LR, van Kaam AH, Kohse F, Veltkamp F, Bos LD, de Beer FM et al (2019) Age-dependent differences in pulmonary host responses in ARDS: a prospective observational cohort study. Ann Intensive Care 9(1):55

33. Bloem LJ, Manatunga AK, Pratt JH (1996) Racial difference in the relationship of an angiotensin I-converting enzyme gene polymorphism to serum angiotensin I-converting enzyme activity. Hypertension 27(1):62-66
34. Saab YB, Gard PR, Overall AD (2007) The geographic distribution of the ACE II genotype: a novel finding. Genet Res 89(4):259-267

35. Delanghe JR, Speeckaert MM, De Buyzere ML (2020) The host's angiotensin-converting enzyme polymorphism may explain epidemiological findings in COVID-19 infections. Clin Chim Acta 505:192-193

36. Cao J, Naeem M, Noh J-K, Lee EH, Yoo J-W (2015) Dexamethasone phosphate-loaded folate-conjugated polymeric nanoparticles for selective delivery to activated macrophages and suppression of inflammatory responses. Macromol Res 23(5):485-492

37. Cao Y, Li L, Feng Z, Wan S, Huang P, Sun X et al (2020) Comparative genetic analysis of the novel coronavirus (2019-nCoV/ SARS-CoV-2) receptor ACE2 in different populations. Cell Discov 6:11

38. Chen L, Li X, Chen M, Feng Y, Xiong C (2020) The ACE2 expression in human heart indicates new potential mechanism of heart injury among patients infected with SARS-CoV-2. Cardiovasc Res 116(6): 1097-1100 